\title{
L-BAND SPECTROSCOPY WITH MAGELLAN-AO/Clio2: FIRST RESULTS ON YOUNG LOW-MASS COMPANIONS
}

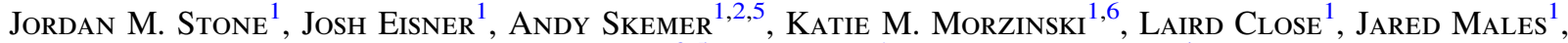 \\ Timothy J. Rodigas ${ }^{3,5}$, Phil Hinz ${ }^{1}$, and Alfio Puglisi ${ }^{4}$ \\ ${ }^{1}$ Steward Observatory, University of Arizona, 933 N. Cherry Ave, Tucson, AZ 85721-0065, USA \\ ${ }^{2}$ Department of Astronomy and Astrophysics, University of California, Santa Cruz, 1156 High St, Santa Cruz, CA 95064, USA \\ ${ }^{3}$ Department of Terrestrial Magnetism, Carnegie Institution of Washington, 5241 Broad Branch Road NW, Washington, DC 20015, USA \\ ${ }^{4}$ INAF-Osservatorio Astrofisico di Arcetri, I-050125, Firenze, Italy \\ Received 2015 December 16; revised 2016 August 4; accepted 2016 August 4; published 2016 September 21
}

\begin{abstract}
L-band spectroscopy is a powerful probe of cool low-gravity atmospheres: the $\mathrm{P}, \mathrm{Q}$, and $\mathrm{R}$ branch fundamental transitions of methane near $3.3 \mu \mathrm{m}$ provide a sensitive probe of carbon chemistry; cloud thickness modifies the spectral slope across the band; and $\mathrm{H}_{3}^{+}$opacity can be used to detect aurorae. Many directly imaged gas-giant companions to nearby young stars exhibit L-band fluxes distinct from the field population of brown dwarfs at the same effective temperature. Here we describe commissioning the L-band spectroscopic mode of Clio2, the 1-5 $\mu \mathrm{m}$ instrument behind the Magellan adaptive-optics system. We use this system to measure L-band spectra of directly imaged companions. Our spectra are generally consistent with the parameters derived from previous near-infrared spectra for these late M to early L type objects. Therefore, deviations from the field sequence are constrained to occur below $1500 \mathrm{~K}$. This range includes the L-T transition for field objects and suggests that observed discrepancies are due to differences in cloud structure and $\mathrm{CO} / \mathrm{CH}_{4}$ chemistry.
\end{abstract}

Key words: binaries: close - stars: atmospheres - stars: low-mass

\section{INTRODUCTION}

Low-gravity atmospheres, like those of young gas-giant planets, display distinct characteristics compared to their higher-gravity counterparts, even at the same effective temperatures (Martin et al. 1996; Marois et al. 2008; Bowler et al. 2010; Barman et al. 2011b; Currie et al. 2011; Marley et al. 2012; Skemer et al. 2012). Both cloudiness and disequilibrium chemistry are enhanced as a result of the prevailing low pressure in the atmospheres of the young low-mass objects: clouds because the photosphere is beneath the temperature-pressure location where refractory elements begin to condense to liquid and solid phases; and dis-equilibrium chemistry because the longer chemical timescales implied by the low pressure (and density) cannot keep up with vertical mixing timescales that deliver warm material from deeper layers (e.g., Hubeny \& Burrows 2007; Barman et al. 2011a; Marley et al. 2012).

As demonstrated in the cases of $2 \mathrm{M} 1207 \mathrm{~b}$ (Chauvin et al. 2004) and HR 8799 b, c, and d (Marois et al. 2008), disequilibrium chemistry, vertical mixing, and cloudiness are important factors in understanding the observed features of cool young/low-gravity atmospheres (Skemer et al. 2011; Barman et al. 2011a, 2011b). Clouds and dis-equilibrium $\mathrm{CH}_{4} / \mathrm{CO}$ chemistry have a large effect on L-band spectra because the P-, Q-, and R-branch methane bandheads trace the abundance of $\mathrm{CH}_{4}$, and cloudiness affects the general slope of the spectrum (Yamamura et al. 2010; Skemer et al. 2014). L-band measurements augmenting near-IR $(\mathrm{Y}, \mathrm{J}, \mathrm{H}$, and $\mathrm{K}$ band) spectra can also help to break degeneracies (e.g., between metalicity and gravity) when performing fits to model atmospheres (Stephens et al. 2009; Skemer et al. 2015).

\footnotetext{
5 Hubble Fellow.

6 Sagan Fellow.
}

Atmospheric properties are crucial for understanding the nature and origin of directly imaged companions. The HR 8799 system contains four directly imaged companions with a nonhierarchical orbital architecture that closely resembles a planetary system, albeit much more massive then typical (Marois et al. 2008, 2010). Yet with uncertain companion masses $\left(\sim 10 M_{\text {Jup }}\right)$ it is possible (though unlikely) that at least some of the companions exceed the $\sim 12 M_{\text {Jup }}$ minimum mass for deuterium burning. ${ }^{7}$ PSO J318.5-22, a $\sim 6 M_{\text {Jup }}$ isolated member of the $\beta$ Pic moving group, occupies the same locus of color-magnitude space as HR 8799 b, c, d, e, and 2M 1207 b, but this object is not a planet because it was likely born in isolation (Liu et al. 2013b).

PSO J318.5-22 and other low-mass brown dwarfs in the field appear to form through an extension of the star-formation process to very low masses (Luhman 2012). Similarly, 2M 1207 b likely formed as an extension of the binary-star formation process (Lodato et al. 2005). The orbital architecture of the HR 8799 system suggests a distinct formation mechanism, such as core-accretion or gravitational-instability in a circum-stellar disk (Kratter et al. 2010). Thus it appears nature is capable of producing fundamentally different objects, with distinct formation processes that nevertheless have overlapping "planetary" masses.

Spectroscopy constrains the formation mechanisms of directly imaged companions by revealing the composition of their atmospheres (Konopacky et al. 2013; Barman et al. 2015). If companion atmospheres gain a significant fraction of their mass via the accretion of solids in a disk, then they should show increased metalicity. The core-accretion process for gasgiant planet formation can produce metal enhanced planetary atmospheres compared to the host star (Pollack et al. 1996).

\footnotetext{
The current IAU definition of a planet is an object below the deuterium burning limit that is gravitationally bound to a more massive primary. See Chabrier et al (2014, p. 619) for a discussion of some of the issues with this definition.
} 
Table 1

Observing Log

\begin{tabular}{|c|c|c|c|c|c|c|}
\hline Target & Date & $\begin{array}{l}\text { Plate Scale } \\
\left(\text { mas pix }^{-1}\right)\end{array}$ & $\begin{array}{l}\text { Tot. Int. } \\
\text { (s) }\end{array}$ & $R^{\mathrm{a}}$ & Guidestar mag ${ }^{\mathrm{b}}$ & Comments \\
\hline CD-35 2722 B & 2014 Apr 07 & 15.9 & 280 & $50-200$ & $m_{I}=9 \mathrm{~s}$ & \\
\hline TWA 5 B & 2014 Apr 07 & 15.9 & 2240 & $50-200$ & $m_{I}=9.1$ & \\
\hline HD 138575 & 2014 Apr 07 & 15.9 & 840 & $50-200$ & $m_{V}=7$ & A0V telluric calibrator \\
\hline HD 32007 & 2014 Nov 14 & 25.7 & 540 & $150-300$ & $m_{V}=8.5$ & B9 telluric calibrator \\
\hline AB Pic b & 2014 Nov 14 & 25.7 & 3690 & $150-300$ & $m_{R}=9$ & \\
\hline$\eta$ Tel B & 2014 Nov 15 & 25.7 & 720 & $150-300$ & $m_{R}=5$ & \\
\hline $2 \mathrm{M} 0103(\mathrm{AB}) \mathrm{b}$ & 2014 Nov 15 & 25.7 & 1080 & $150-300$ & $m_{I}=12.9$ & guidestar is $\sim 0$ ". 2 binary \\
\hline HD 10553 & 2014 Nov 15 & 25.7 & 360 & $150-300$ & $m_{V}=6.6$ & A3V telluric calibrator \\
\hline
\end{tabular}

Notes.

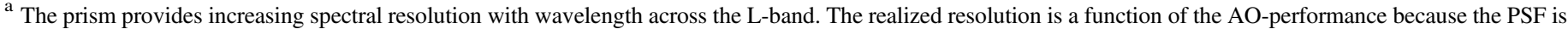
smaller than the slit.

${ }^{\mathrm{b}}$ For our companion sources we used the primary stars as the AO-guidestar. The wavefront sensor uses a non-standard filter covering the R and I bands.

Gravitational fragmentation of a massive protoplanetary disk can also result in a metal enriched atmosphere (Boss 1997; Boley et al. 2011). Furthermore, because of the radial temperature gradient in protoplanetary disks, certain chemical species will be removed from the gas phase at radii corresponding to their freeze-out temperatures. This will result in distinct elemental ratios in the planetary atmosphere which may be diagnostic of the radial position at which a given planet formed in a disk (e.g., Öberg et al. 2011). On the other hand, if a companion is produced via gravitational fragmentation of the initial dense molecular cloud core, no large compositional differences are expected. The utility of spectroscopy for discriminating between formation modes relies on compositional differences being manifest in the atmospheres of directly imaged companions and may be affected by different assumptions regarding how material from deep within an object can be transported to the outer envelope (e.g., Thiabaud et al. 2015).

The Magellan adaptive-optics system (MagAO; Close et al. 2013), with its adaptive secondary mirror, provides low-background L-band images at the diffraction limit of the $6.5 \mathrm{~m}$ Magellan Clay telescope (providing a point-spread function (PSF) with a width of 121 mas). The mid-infrared camera, Clio2 (like Clio, Sivanandam et al. 2006; Hinz et al. 2010, but with an upgraded HAWAII-1 array) that operates behind the MagAO system, is equipped with a prism that yields increasing spectral resolution from $R \sim 50-300$ across the L-band. Given the sensitive high-spatial resolution capabilities of MagAO, Clio2 spectroscopy is a powerful tool for providing the L-band spectroscopic constraints necessary for a complete understanding of directly imaged low-mass companion atmospheres. MagAO/Clio2 complements ongoing near-IR surveys for the detection and spectral characterization of new companions, such as the Gemini Planet Imager Exoplanet Survey (Macintosh et al. 2015), and surveys with the SPHERE instrument at the Very Large Telescope (Beuzit et al. 2008), neither of which are sensitive in the L-band.

In this paper, we establish the L-band spectroscopic mode of $\mathrm{MagAO} / \mathrm{Clio} 2$ and report first results on low-mass directly imaged companions. In Section 2 we describe our sample of targets and summarize near-IR constraints on the nature of their atmospheres. We also describe our data collection and reduction approach for the new mode. In Section 3 we summarize our L-band spectroscopic results for the sample.
Finally, in Section 4 we discuss the implications of our findings.

\section{OBSERVATIONS AND REDUCTION}

Data for this work were collected during two observing runs in 2014. The first was from 2014 April 5 to 6, and the second was from 2014 November 13 to 15 . A summary of our observing $\log$ s is presented in Table 1. Our data collection and calibration approach is described below.

\subsection{Target Selection and Parameters from Literature}

Our target list, summarized in Table 2, was selected to include nearby young stars with low-mass companions visible from Las Campanas Observatory (declination $\lesssim 20^{\circ}$ ). Given the sensitivity of the MagAO wavefront sensor and of Clio2, we also selected targets to have guidestar I-band magnitudes $\lesssim 13$ and companion L-band magnitudes $\lesssim 14$ to facilitate obtaining adequate signal to noise in less than one night of observing. We focused on targets with well characterized near-IR spectra for direct comparison to our results. One of our targets, 2MASS J01033563-5515561 $(\mathrm{AB}) \mathrm{b}$ (hereafter 2M 0103(AB) b), which orbits a 0". 2 M6-M6 binary-star system, does not have near-IR spectra available in the literature. We added this target to bolster our low-mass sample and to test the performance of MagAO while guiding on a close nearly equal-magnitude binary.

\subsection{Instrumental Setup}

The MagAO system is built around a 585 actuator adaptive secondary mirror that minimizes the number of optical elements necessary for correcting the blurring effects of Earth's atmosphere. Compared to alternative implementations that position an adaptive element at a re-imaged pupil plane, adaptive-secondary systems provide higher throughput and lower thermal background (Lloyd-Hart 2000). The system provides correction for up to 300 modes at up to $989 \mathrm{~Hz}$. The pyramid wavefront sensor used by MagAO facilitates easy adjustments both in the number of modes corrected and the loop speed to help keep the AO loop locked on faint guidestars or in periods of poor seeing.

The prism in Clio2 provides increasing spectral resolution with wavelength. At $\mathrm{J}$ band the typical resolution is $R \sim 8$ and at $\mathrm{M}$ band it is $R \sim 500$. We used a 260 mas slit to minimize the bright mid-infrared sky emission in our spectra. For 
Table 2

Summary of Target Information from the Literature

\begin{tabular}{|c|c|c|c|c|c|c|c|c|c|}
\hline \multirow[b]{2}{*}{ Name } & \multirow[b]{2}{*}{$\begin{array}{l}\text { Dist. } \\
\text { (pc) }\end{array}$} & \multirow[b]{2}{*}{$\begin{array}{l}\text { Sep. } \\
\left({ }^{\prime \prime}\right)\end{array}$} & \multirow[b]{2}{*}{ Sp. Type } & \multicolumn{2}{|c|}{ NIR Template Fit ${ }^{\mathrm{a}}$} & \multirow[b]{2}{*}{ Assoc. } & \multirow[b]{2}{*}{$\begin{array}{c}\text { Est. Mass } \\
\left(M_{\mathrm{Jup}}\right)\end{array}$} & \multirow[b]{2}{*}{$\begin{array}{c}\text { Est. Age } \\
\text { (Myr) }\end{array}$} & \multirow[b]{2}{*}{ References } \\
\hline & & & & $\begin{array}{l}T_{\text {eff }} \\
(\mathrm{K})\end{array}$ & $\begin{array}{c}\log (g) \\
\left(\mathrm{cm} \mathrm{s}^{-2}\right)\end{array}$ & & & & \\
\hline$\eta$ Tel B & 48 & 4.2 & M7-9 & $2600 \pm 100$ & $4 \pm 0.5$ & $\beta \mathrm{Pic}$ & $\sim 30$ & $\sim 12$ & $5,12-14$ \\
\hline TWA 5 B & 50 & 2 & $\mathrm{M} 8.5 \pm 0.5$ & $2500 \pm 100$ & $4 \pm 0.5$ & TW Hya & $\sim 20$ & $\sim 10$ & $5,8-11$ \\
\hline CD-35 2722 B & 21 & 3.1 & L3-L4 & 1700-1900 & $4.5 \pm 0.5$ & AB Dor & $\sim 13$ & $\sim 50$ & $2,3,15$ \\
\hline $\mathrm{AB} P i c \mathrm{~b}$ & 46 & 5.5 & $\mathrm{~L} 0 \pm 1$ & $1800_{-200}^{+100}$ & $4.5 \pm 0.5$ & Tuc-Hor & $\sim 12$ & $\sim 30$ & 4-7 \\
\hline $2 \mathrm{M} \mathrm{0103(AB)} \mathrm{b}$ & 47 & 1.7 & $\mathrm{~L}$ & $\ldots$ & $\ldots$ & Tuc-Hor & $\sim 13$ & $\sim 30$ & 1,7 \\
\hline
\end{tabular}

Note.

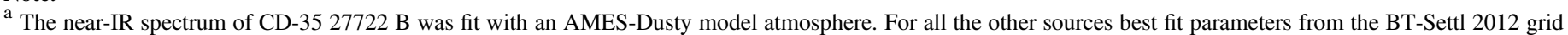
are reported.

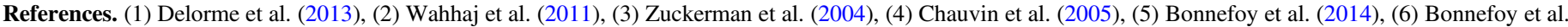

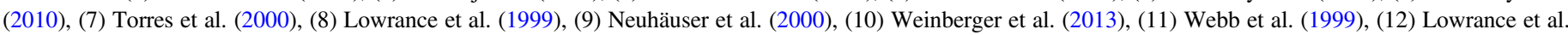
(2000), (13) Neuhäuser et al. (2011), (14) Zuckerman et al. (2001), (15) Allers \& Liu (2013).

observations with the PSF smaller than the slit, the size of the PSF is the relevant parameter for determining the spectral resolution. Thus, our observed spectral resolution changes with AO performance and wavelength. During the two runs reported here, we realized $R=50-200$ and $R=150-300$ across the L-band.

Due to the very different dispersions in the near- and mid-IR, there are different timescales for detector saturation in each band. In order to reduce practical complications related to saturation and cross-talk on the Clio 2 detector, we observed through a blocking filter that only passes light from $\sim 2.8$ to $\sim 4.2 \mu \mathrm{m}$.

Two camera lenses provide Clio2 with two plate scales: a coarse mode with 27.5 mas pixel $^{-1}$, and a fine mode with 15.9 mas pixel $^{-1}$ (Morzinski et al. 2015). As part of our effort to define best practices for the use of the MagAO/Clio2 spectroscopy mode, we used a different plate scale for each of our runs. This also accommodated the observation of wider binaries. The optics used to set the plate scale modify the position of the spectral trace on the Clio 2 detector. The fine mode situates the trace on the left side of the detector, and the coarse mode positions the trace on the right side, which has more high dark current pixels and produces noisier spectra as a result.

\subsection{Data Acquisition}

We used the fine plate scale and observed both TWA 5 and CD-35 2722 during our first run. For our second run, we used the coarse plate scale and observed $\eta$ Tel B, AB Pic b, and $2 \mathrm{M} \mathrm{0103(AB)} \mathrm{b.} \mathrm{We} \mathrm{saved} \mathrm{frames} \mathrm{differently} \mathrm{for} \mathrm{each} \mathrm{run,}$ saving single images per nod position during the first run and multiple images per nod during the second run. This difference slightly changes our reduction procedure for each dataset, as discussed below.

For each object, we aligned the binary position angle of our targets to be parallel to the Clio 2 slit so that we could collect spectra of both primary and companion sources simultaneously. This orientation allows us to use the primary star as a telluric calibrator for the companion. Because the slit position and angle are not repeatable, and because Clio2 does not have a slit viewer, we developed the following alignment procedure.

First, after acquiring our targets with the slit and prism out of the beam, we noted the position of the primary star and the position angle of the companion. Next, we introduced the slit and nudged the telescope horizontally until the primary star could be seen in the center. We then measured the angle of the slit on the detector and calculated the necessary slit rotation offset to align the target PA with the slit. The calculated rotation offset was implemented in small steps, $\lesssim 5^{\circ}$, without opening the AO loop. By keeping the AO loop closed on the primary star while we offset the rotator to align our targets, we ensured that the star remained on the rotation axis, stationary in the slit.

Once both objects were aligned in the slit, we introduced the prism. In order to track variable background sky emission, we nodded our targets every 1-3 minutes. When we could, we nodded along the slit so that we were always integrating on the target. At some rotation angles, when the entire weight of the wavefront sensor apparatus rested on just one motor, we had to nod off the slit because the motors were insufficiently powered to provide the desired nod vector. Currently both the previous weaker 1.6 Amp motors have been replaced by $3.0 \mathrm{Amp}$ motors to hold the full weight.

On 2014 November 15, we observed $\eta$ Tel during twilight because the source was setting. Since the sky was bright in the optical, we were unable to perform the normal telescope collimation steps. We ran the AO loop closed with only 10modes for five minutes in order to offload as much focus to the telescope as possible, and then increased the number of modes to begin observing. Twilight background light on the wavefront sensor still limited the accuracy of AO corrections and poor AO-performance was realized for this source.

\subsection{Preliminary Reduction}

Our data reduction process began by subtracting nod pairs. For the data from April this was simply an A-B and a B-A subtraction, where A and B represent our two nod positions. For the data from November, when we saved 6 images per nod position, we did $\mathrm{A}_{i}-\operatorname{median}(\mathrm{B})$ and $\mathrm{B}_{i}-\operatorname{median}(\mathrm{A})$, $i=1-6$. We then rotated each image to produce horizontal spectral traces. For the November data, we also applied a vertical shift to align each of the 6 images per nod, and coadded them. Typical rotations were $\sim 1.3$ and typical vertical offsets were $\lesssim 0.1$ pixels.

We created a master spectral trace for each object by combining the traces from each nod sequence. For our April observations we combined the traces using a simple mean. For our November observations, since we saved multiple images 


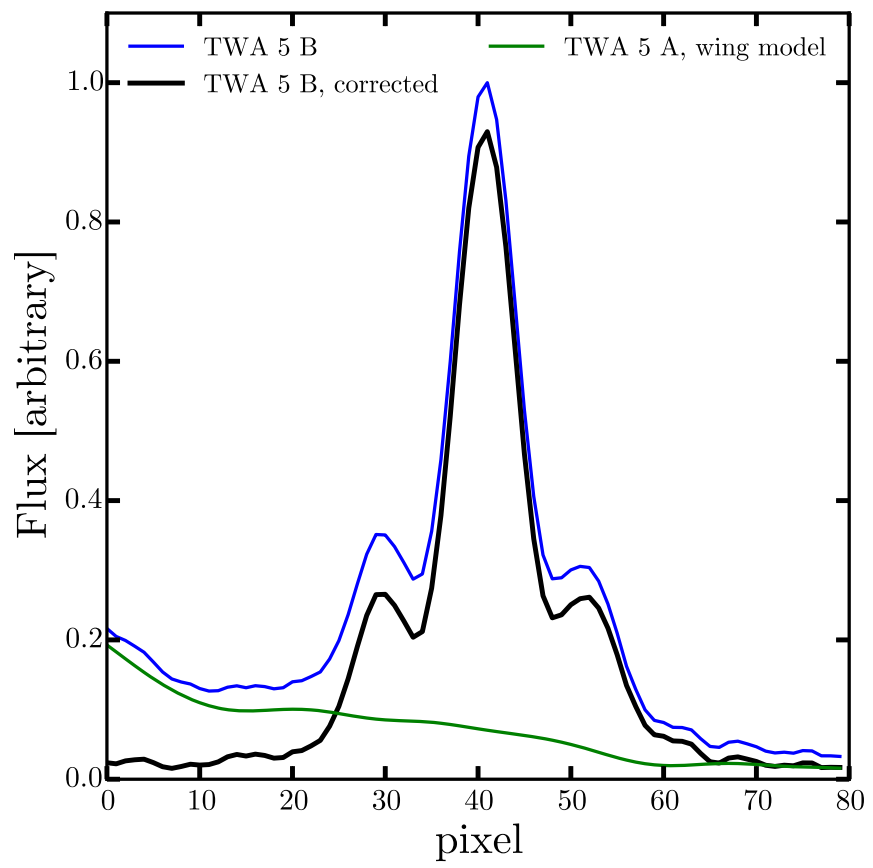

Figure 1. A slice through the spatial dimension of the trace of TWA5 B. Light from the unresolved binary host influences the left shoulder. We removed the influence of the primary star using a model constructed by scaling the columns of a telluric calibrator trace (see text).

per nod position, we were able to measure the image variance in each pixel in each nod, so we derived a weight-image for each nod inversely proportional to the image variance before combining. For all of our sources we also bootstrapped the stacking procedure, randomly selecting individual frames (with replacement), before stacking. This provides a variance image for use in determining optimal weights for spectral extraction (see below), and the raw data for bootstrapping the extraction, wavelength calibration, and telluric correction steps necessary for generating errorbars for our spectra.

For close companions, spectra may be contaminated by the wings of the primary if the primary flux at the position of the companion is larger than the background noise. TWA $5 \mathrm{~B}$ and

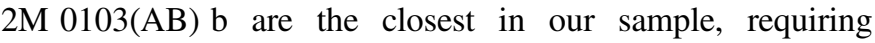
attention to this effect. To remove the light of the primary from the spectrum of the secondary, we created a model of the primary star trace using a high signal-to-noise image of a telluric calibrator spectrum observed just after observing the target. We rotated and shifted the telluric trace to overlap the trace of the primary. We then scaled each column of the telluric image so that the pixels in the core and first Airy ring best-fit the corresponding pixels in the image of the primary. This provided us with a model of the trace of the primary without the secondary. We smoothed this model with a circular Gaussian kernel with $\sigma=4$ pixels, and then subtracted it from the image before extracting the companion spectrum. In Figure 1 we show the average spatial profile of TWA 5 B before and after correction.

For the 2M 0103 system, we achieved much lower signal-tonoise than for TWA 5. Therefore our model trace was also noisy. Since the magnitude of the correction for 2M 0103 (AB) b was much smaller than the noise, we decided not to subtract a noisy model from the images before extraction.

\subsection{Spectral Extraction}

We used the wavelength dependent spatial profiles of primary stars and our bootstrap generated variance maps to define optimal weights (Horne 1986) for extraction. We found that including the first Airy ring in the spatial profile was important to avoid fringing in our spectra because spectral traces exhibit a fringe pattern where the core and Airy rings oscillate out of phase. For 2M 0103(AB) b, the circumbinary source, the primary spatial profile was corrupted by light from the nearby secondary. In this case we used the spatial profile of the telluric calibrator star observed shortly after we observed the target.

\subsection{Wavelength Calibration}

We derived our wavelength calibration based on the location of telluric absorption features in our spectra. First, we verified that the wavelength solution does not change-modulo horizontal offsets-with position on the detector. Then, for each night, we shifted and stacked all the telluric calibrator spectra using an inverse variance weighted mean to create a high signal-to-noise template spectrum of the telluric absorption.

To identify telluric absorption features in our stacked telluric spectra, we used a high-resolution ATRAN (Lord 1992) synthetic transmission spectrum tuned for Cerro Pachon and made available by the Gemini Observatory. ${ }^{8}$ Our approach was iterative, first smoothing the synthetic transmission spectrum to match a best-guess of the linearly increasing spectral resolution delivered by the instrument and then comparing the smoothed synthetic spectrum to our observed spectrum to derive a second-order polynomial transformation to take pixel position to wavelength. We then performed a two-parameter grid search to better identify the appropriate linearly increasing resolution parameters for the synthetic spectrum. This process was repeated until the wavelength solution and spectral resolution parameters converged. Figure 2 shows our stacked telluric calibrator spectra for each run and the ATRAN telluric transmission spectrum smoothed using the best-fit linearly increasing resolution parameters.

The best fit for 2014 April 7 (15.9 mas pixel $^{-1}$ mode) was $R=50-200$. The best fit for both 2014 November 14 and 15 (25.7 mas pixel ${ }^{-1}$ mode) was $R=150-300$. The functional forms of the wavelength solutions for each run, modulo horizontal pixel offsets, are

$$
\lambda(x)=\left(-2.503 \times 10^{-6}\right) x^{2}+(0.004801) x+2.822,
$$

for our April run, with an rms scatter of $0.012 \mu \mathrm{m}$, and

$$
\lambda(x)=\left(-3.634 \times 10^{-6}\right) x^{2}+(0.00548) x+2.786,
$$

for our November run, with an rms scatter of $0.007 \mu \mathrm{m}$. We wavelength calibrated the science target spectra by fitting for the best horizontal offset between target and calibrator, using a cubic interpolation scheme to perform the shift.

Two of our targets, CD-35 $2722 \mathrm{~B}$, and 2M 0103(AB) b, did not have high enough signal-to-noise to provide an adequate fit for the best horizontal offset. For these sources, we calculated the appropriate horizontal offset using the measured rotation angle of the slit and the separation from

\footnotetext{
8 http://www.gemini.edu/sciops/telescopes-and-sites/observing-conditionconstraints/ir-transmission-spectra
} 

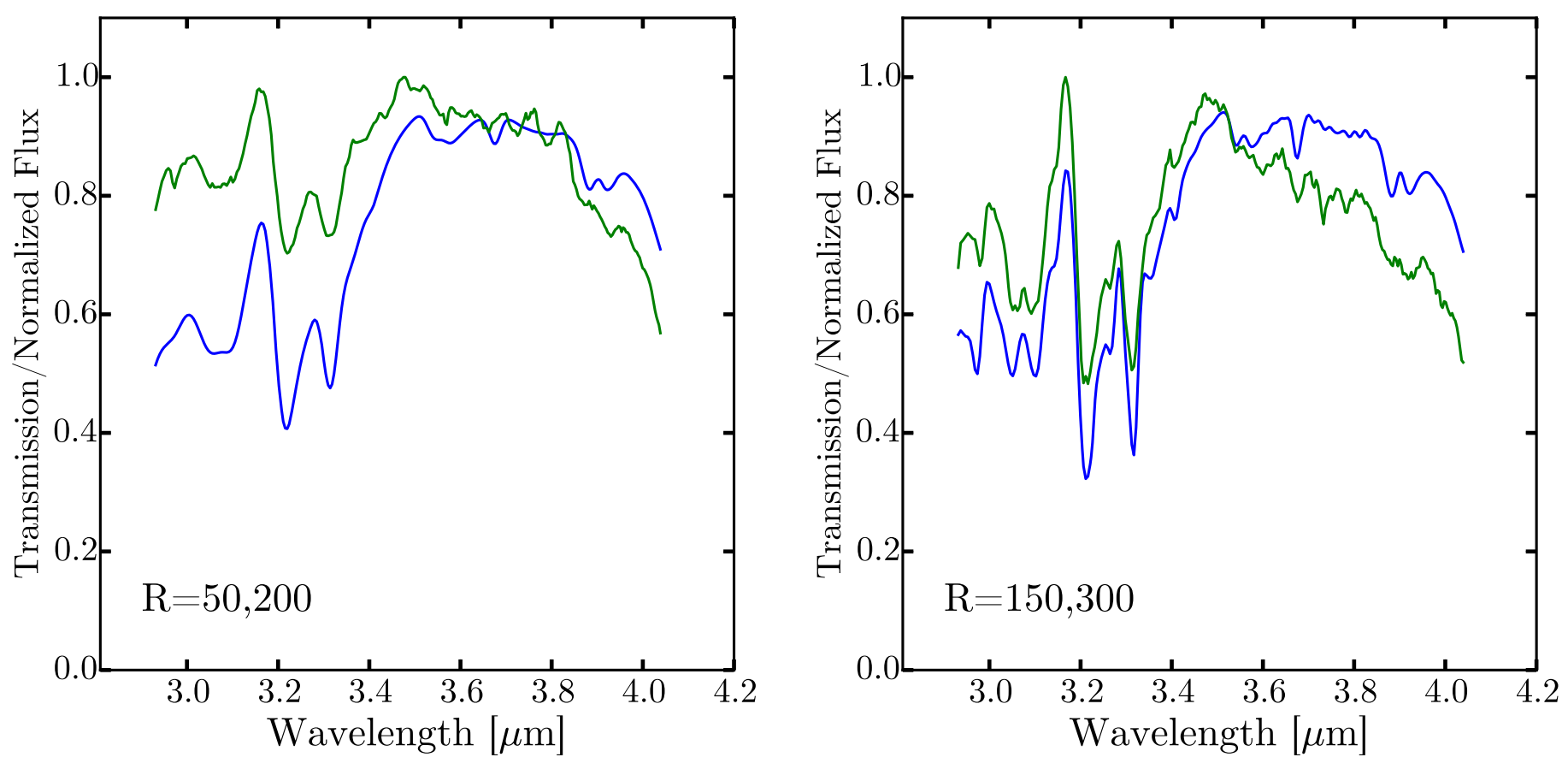

Figure 2. Smoothed ATRAN synthetic transmission spectra, demonstrating our spectral resolution during each run (blue curves). We also show a weighted average of our observed telluric calibrator spectra (green curves). The left side corresponds to our April run, and the right side corresponds to our November run.

the primary. We verified using our other targets that this approach yields the correct offset to within 1-2 pixels.

\subsection{Telluric Calibration}

Our telluric calibration strategy used primaries to correct companions, and A-stars to correct primaries. We started by correcting primaries. We divided by the A-star spectrum obtained closest in time to a target spectrum and then multiplied by $\lambda^{-4}$ to correct for the intrinsic blackbody shape of the hot calibrator (this introduces $<2 \%$ error). Since most primaries have a more complicated intrinsic spectrum, before correcting companion spectra we fit for the best synthetic atmosphere spectrum from the grids of BT-Settl atmospheres (solar metallicity models from both the 2012 and 2015 grids were used; Allard et al. 2012; Baraffe et al. 2015). We smoothed the synthetic spectra using the same linearly increasing resolution parameters that we derived during our wavelength calibration process. We calibrated companions by dividing by primary spectra and then multiplying by the best-fit model for the primary. In two cases, $\eta$ Tel (primary spectral type A0V; Torres et al. 2006) and CD-35 2722 (primary spectral type M1V; Torres et al. 2006), we did not obtain an adequate telluric calibrator spectrum. In these cases we used spectral types from the literature to select the appropriate model for the intrinsic shape of the primary spectrum, using the spectral-type- $T_{\text {eff }}$ relationship of Pecaut \& Mamajek (2013).

\section{RESULTS}

\subsection{Model Atmosphere Fits}

For each object we determined the best fit model atmosphere from the latest BT-Settl grid (Baraffe et al. 2015). Many of our companions have had BT-Settl 2012 models (Allard et al. 2012) fit to their near-IR spectra, so we also fit to that grid to facilitate a direct comparison. We used models with

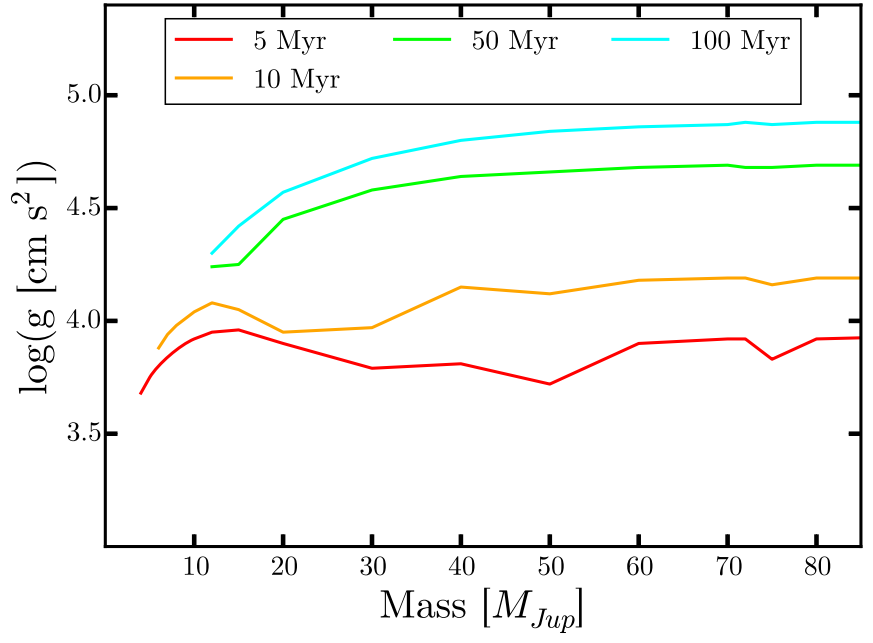

Figure 3. Predicted surface gravity as a function of mass from the BT-Settl 2015 models. Each curve represents a different snapshot in age, with the lowest surface gravities corresponding to $1 \mathrm{Myr}$, and the highest gravities corresponding to $100 \mathrm{My}$. Substellar objects with masses $\geqslant 2 M_{\mathrm{Jup}}$ and ages older than 1 Myr should have $\log (g)>3$. Likewise objects less massive than $80 M_{\text {Jup }}$ and younger than 100 Myr should have $\log (g)<5$.

effective temperatures ranging from 1200 to $7000 \mathrm{~K}$, restricting ourselves to models with solar metallicity and with surface gravity between $\log (g)=3.5$ and $\log (g)=5.0$. Figure 3 illustrates how we chose this range of gravities. At ages greater than $5 \mathrm{Myr}$, even $2 M_{\text {Jup }}$ objects should have surface gravity greater than $\log (g)=3.5$. Likewise, even $100 \mathrm{Myr}$ old brown dwarfs at $80 M_{\text {Jup }}$ should have surface gravity less than $\log (g)=5.0$. These restrictions fold in our prior knowledge on the age of our targets into the fitting process. After smoothing model spectra to the resolution of Clio2, fitting was done via $\chi^{2}$ minimization using the inverse of our bootstrap errorbars as weights. Our fits ignored the spectral regions between 


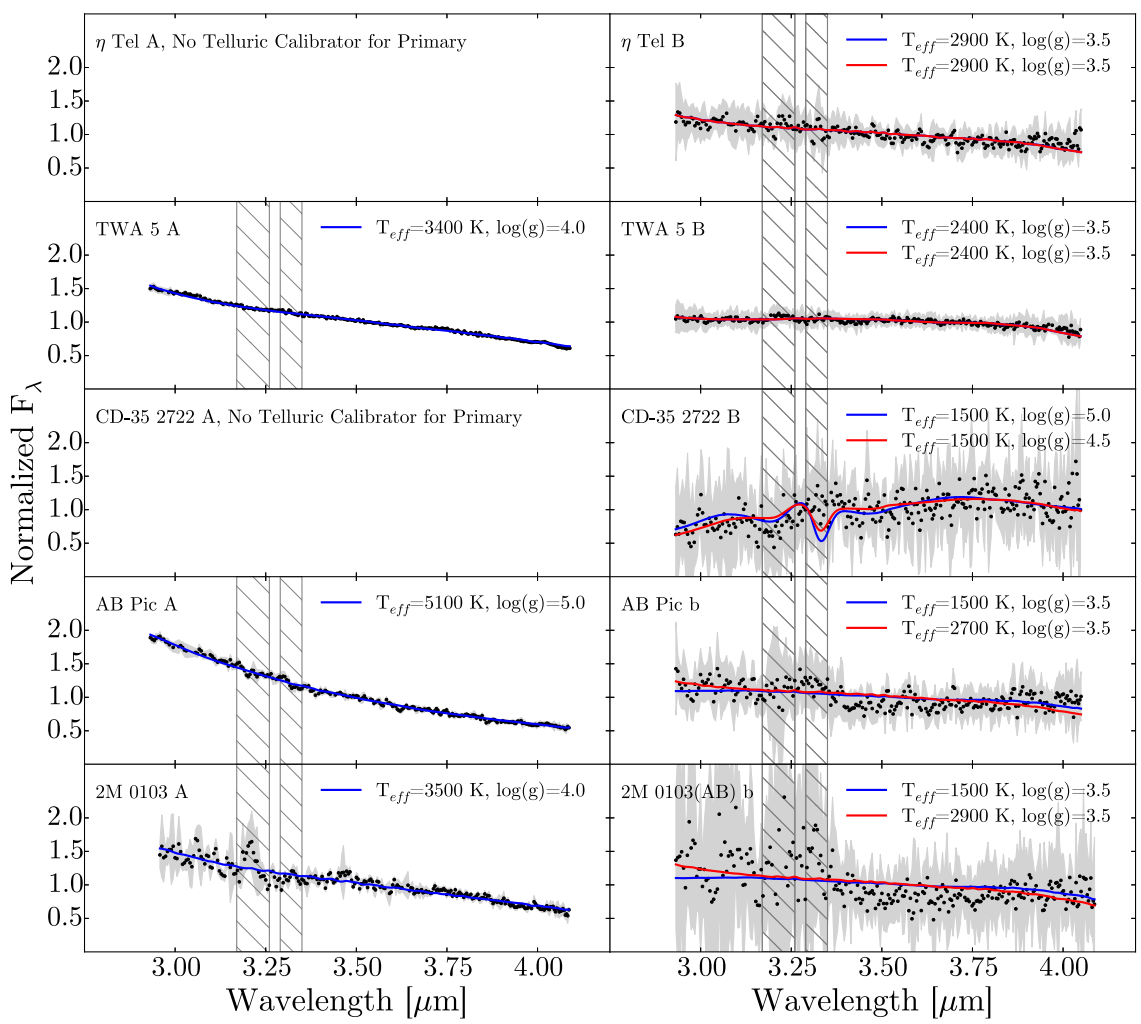

Figure 4. L-band spectra of young low-mass companions and their primary stars. Black points indicate measurements and the gray swath shows the 1- $\sigma$ confidence region after scaling bootstrap errors by a factor of 5 to account for flat-field effects and fixed pattern noise (see Section 3.1). We also show model atmosphere fits to our spectra. In blue, we show the best fit from the BT-Settl 2015 grid, and in red we show the best fit from the BT-Settl 2012 grid. For our primaries, there were no differences in appearance of best-fit models from either grid. Hatched regions near 3.2 and $3.3 \mu \mathrm{m}$ indicate the location of strong telluric absorption features in our data. These regions were not considered during our model fitting. For two of our primaries, we did not collect appropriate telluric calibrator spectra, so we do not show their uncorrected spectra. We are still able to correct their secondaries because we assumed previously reported spectral types to determine their intrinsic spectral shape.

$3.17-3.26 \mu \mathrm{m}$ and $3.29-3.35 \mu \mathrm{m}$ because these regions are affected by strong telluric absorption and are most susceptible to calibration errors. The best fits for each object are shown in Figure 4.

The range of allowed model atmosphere parameters consistent with our data depends on an accurate estimation of our observational uncertainty. As discussed in Section 2.4, we measured the magnitude of random errors in our data using the bootstrap method, but fixed-pattern noise-from flat field effects and bad pixels - adds systematic error to our spectra. To account for this, we scaled up the size of our errorbars using a single empirically determined scale factor for every spectrum. This scale factor was determined as follows. We selected our highest signal-to-noise spectra, excluding the unresolved binary sources TWA $5 \mathrm{~A}$ and $2 \mathrm{M} 0103 \mathrm{AB}$. This left us with $\mathrm{AB}$ Pic $\mathrm{A}, \eta \mathrm{Tel} \mathrm{B}$, and TWA $5 \mathrm{~B}$. We then scaled the size of the errorbars for each source until the best-fit model atmosphere provided a reduced $\chi^{2}$ of 1 . We found scale factors ranging from 3.8 to 5 . We adopted the value of 5 corresponding to the scale factor for $\mathrm{AB}$ Pic A because it is more conservative (larger errors lead to larger allowed ranges for model parameters) and because it corresponds to a hotter, more massive star where atmospheric model parameters are more mature and better tested. The size of our scaled uncertainties are indicated with gray swaths in Figure 4.

In Figure 5, we show $\chi^{2}$ as a function of temperature and gravity for each of our sources. The vertical extent of each plot shows $\Delta \chi^{2}=11.8$ above the minimum-this is the $99.7 \%$ (3- $\sigma$ ) likelihood interval for the case of Gaussian distributed noise. We also indicate the $68 \%(1-\sigma)$ confidence level with a dashed line.

Table 3 lists the results of our model atmosphere fitting. We indicate both the best fit-model atmosphere parameters taken from the grid, as well as the weighted mean atmospheric parameters calculated according to

$$
\bar{m}=\frac{\sum_{i} W_{i} m_{i}}{\sum_{i} W_{i}}
$$

where the weight $W_{i}$ for each model $m_{i}$ is given by

$$
W_{i}=e^{-0.5 \chi^{2}} .
$$

We followed Burgasser et al. (2010) in calculating sided variance estimates for our parameters, using

$$
\sigma_{m \pm}=\frac{\sum_{i \pm} W_{i}\left(m_{i}-\bar{m}\right)^{2}}{\sum_{i \pm} W_{i}}
$$

where the sum is calculated using only parameters above $(+)$ or below (-) the mean values. For AB Pic b and 2M01013(AB) b the $\chi^{2}$ curves in Figure 5 show two minima with similar values. This will result in very large weighted variances.

The 2015 and 2012 grids provide mostly similar fits to our spectra, and these fits reveal atmospheric parameters consistent 

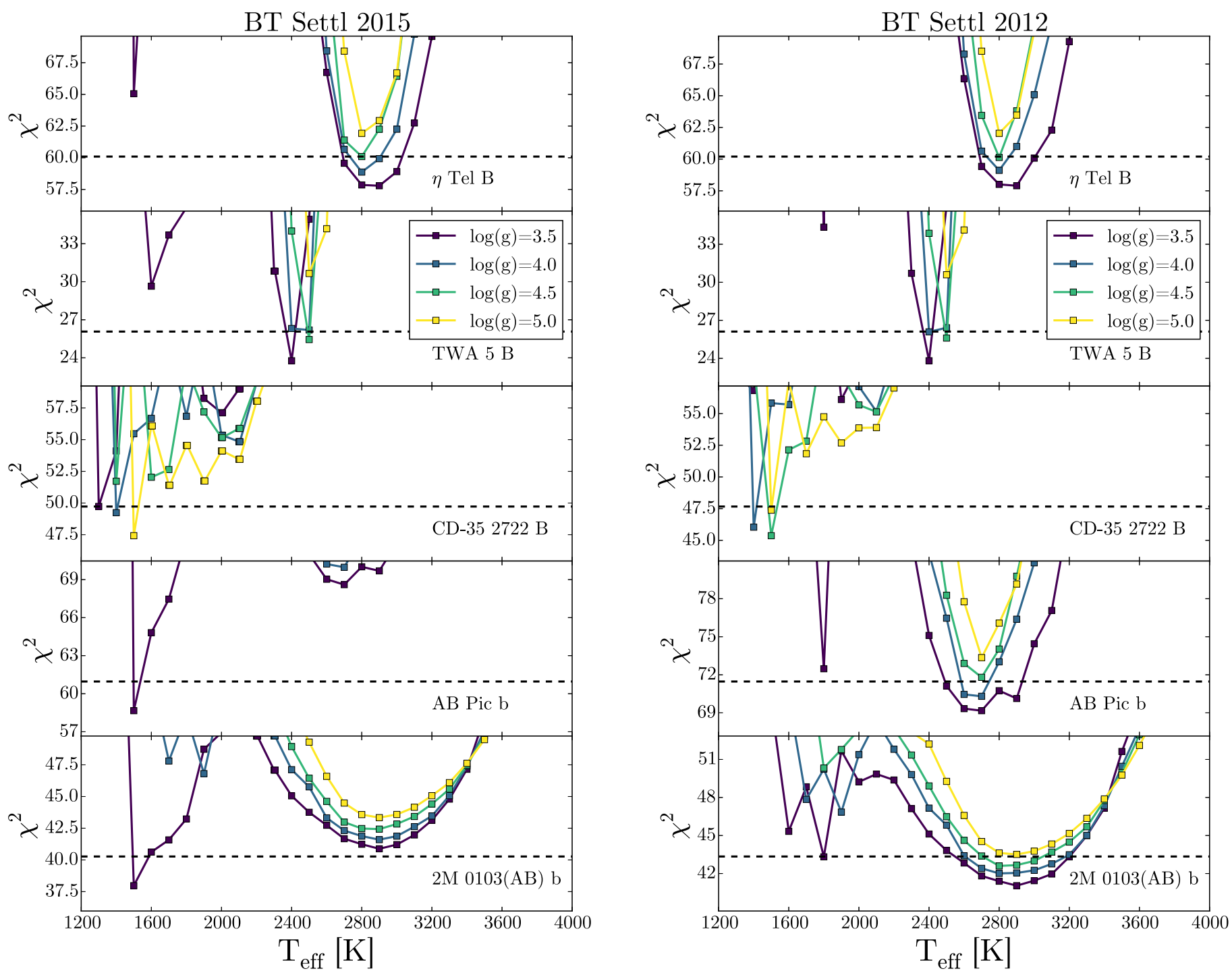

Figure 5. Effective temperature vs. $\chi^{2}$ for each of our secondary spectra. Models with different $\log (g)$ have been plotted with different colors. The best fitting models are shown in Figure 4. The left panel shows the fitting results for the BT Settl 2015 grid and the right panel shows the results for the BT Settl 2012 grid. The vertical extent of each plot reaches $\Delta \chi^{2}=11.8$ : the $99.7 \%$ confidence interval in the case of Gaussian errors. The horizontal dashed line in each panel shows $\Delta \chi^{2}=2.3$, the $68 \%$ confidence interval.

Table 3

BT-Settl Model Atmosphere Fits to L-band Spectra

\begin{tabular}{|c|c|c|c|c|c|c|c|c|c|c|}
\hline \multirow[b]{2}{*}{ Target } & \multicolumn{4}{|c|}{ BT-Settl 2015} & \multicolumn{4}{|c|}{ BT-Settl 2012} & \multicolumn{2}{|c|}{ Fits to NIR Spectra ${ }^{a}$} \\
\hline & $T_{\text {eff,grid }}$ & $\log (g)_{\text {grid }}$ & $T_{\text {eff,mean }}$ & $\log (g)_{\text {mean }}$ & $T_{\text {eff,grid }}$ & $\log (g)_{\text {grid }}$ & $T_{\text {eff,mean }}$ & $\log (g)_{\text {mean }}$ & $T_{\text {eff }}$ & $\log (g)$ \\
\hline$\eta$ Tel B & 2900 & 3.5 & $2838_{-153}^{+114}$ & $<4.6$ & 2900 & 3.5 & $2834_{-80}^{+115}$ & $<4.6$ & $2600 \pm 100$ & $4 \pm 0.5$ \\
\hline TWA 5 B & 2400 & 3.5 & $2410_{-168}^{+90}$ & $<4$ & 2400 & 3.5 & $2431_{-54}^{+70}$ & $<4$ & $2500 \pm 100$ & $4 \pm 0.5$ \\
\hline CD-35 2722 B & 1500 & 5.0 & $1596_{-176}^{+311}$ & $>3.9$ & 1500 & 4.5 & $1497_{-98}^{+136}$ & $4.4_{-0.5}^{+0.3}$ & $1700-1900$ & $4.5 \pm 0.5$ \\
\hline $\mathrm{AB}$ Pic b & 1500 & 3.5 & $1546_{-46}^{+720}$ & $<4.3$ & $2600^{\mathrm{b}}$ & 3.5 & $2664_{-271}^{+136}$ & $<4.1$ & $1800_{-200}^{+100}$ & $4.5 \pm 0.5$ \\
\hline $2 \mathrm{M} 0103(\mathrm{AB}) \mathrm{b}$ & 1500 & 3.5 & $2443_{-592}^{+794}$ & $<4.4$ & $2900^{\mathrm{b}}$ & 3.5 & $2858_{-319}^{+189}$ & $<4.4$ & $\ldots$ & $\ldots$ \\
\hline
\end{tabular}

Notes.

${ }^{a}$ Same as in Table 2, included here for convenience.

b The $\chi^{2}$ surfaces for AB Pic b and 2M 0103(AB) b show two minima with one preferred by fits to the 2012 grid and the other preferred by the 2015 grid. Photometry indicates that the lower-temperature fits are more accurate (see Section 4.3).

with those deduced by fits to $1-2.5 \mu \mathrm{m}$ spectra. Two of our sources, $\mathrm{AB}$ Pic $\mathrm{b}$ and $2 \mathrm{M} 0103(\mathrm{AB}) \mathrm{b}$, are fit by significantly different parameters when using the 2015 and 2012 grid. Figure 5 shows that the difference is due to a change in the shape of model spectra $\sim 1500 \mathrm{~K}$. This can be seen by noting that $\chi^{2}$ values for the hotter best-fit models from the 2012 grid remain unchanged in the 2015 grid, yet significantly better fits are obtained at $1500 \mathrm{~K}$. Both objects exhibit a jump in the 
observed flux level near $3.4 \mu \mathrm{m}$ that persists to the blue-end of the spectrum, resulting in a generally blue slope. We discuss these two targets in more detail in Section 4.3.

Most of our best-fit surface gravities appear at the edge of our restricted range. However, in each case the $1-\sigma$ confidence interval includes the next grid value, except for the 2015-grid fits to $\mathrm{AB}$ Pic $\mathrm{b}$ and $2 \mathrm{M} 0103(\mathrm{AB}) \mathrm{b}$. As discussed above, these sources exhibit an odd feature that is not predicted by the models. Where model fits prefer surface gravities at the edge of our grid, weighted means for this parameter are biased because of the absence of tests performed outside the grid. Thus we report only limits for this parameter for some sources in Table 3.

\subsection{Comparison to Field Dwarf Spectra}

The L-band SEDs of directly imaged extrasolar planets appear distinct from the SEDs of older field dwarfs at the same effective temperatures (Skemer et al. 2014). The discrepancy needs better characterization to provide insight into the physical processes affecting their atmospheres. Comparison to model atmospheres can be helpful in identifying important spectral features, but models are challenged to capture the processes at play in cool atmospheres, particularly in providing accurate representation of clouds (e.g., Marley \& Robinson 2015). Here we compare our spectra of young directly imaged companions to the spectra of field dwarfs from the literature. We use the spectra of M7 through L5 type objects presented in Cushing et al. (2005). We smoothed the template spectra to the resolution of our data and interpolated them to sample the same wavelengths. We then performed the fit by minimizing

$$
\chi_{\text {Emp }}^{2}=\sum_{\lambda} \frac{\left(S_{\lambda, \text { Clio2 }}-S_{\lambda, \text { Spex }}\right)^{2}}{\sigma_{\lambda, \text { Clio } 2}^{2}+\sigma_{\lambda, \text { Spex }}^{2}},
$$

where $S_{\lambda \text {,Clio2 }}$ is our MagAO/Clio2 spectrum and $S_{\lambda \text {,Spex }}$ is the smoothed interpolated field dwarf spectrum from Cushing et al. (2005), and the $\sigma$ 's are the associated errors for each spectrum. We used the same errors for all the field dwarf template spectra, which were determined by requiring that two examples of M9type spectra from Cushing et al. (2005) fit each other well $\left(\chi_{\text {Emp }}^{2}=1\right)$. This helps incorporate the uncertainty in spectral type for our template spectra into our fitting analysis.

Figure 6 shows the best fitting field dwarf spectrum for each of our companion sources. We also overplot the field dwarf spectrum corresponding to the optical/NIR spectral type reported for each of our sources (or the closest sub-type available), except 2M 0103(AB) b, which does not have a NIR spectral type reported in the literature.

In general, the best-fit spectrum and the spectrum selected to match the optical/NIR spectral type are consistent-especially when accounting for the uncertainty in the spectral classification both for our sources and for the field objects. Our data show that for late-M and early $\mathrm{L}$ type spectra, the L-band is not particularly diagnostic of spectral type. For example, the M9 and L3 type dwarf spectra both track the spectrum of TWA 5 B and these are formally both allowed by the data as shown in the right panel of Figure 6, where we quantify the similarities among the fits to different spectral types by showing spectral type versus $\chi_{\text {Emp }}^{2}$ for each of our targets. However, the formal $\Delta \chi^{2}$ analysis should be viewed with caution given the strong influence of systematic uncertainty both in our data and the
Spex data. Previous authors have also noted that L-band spectra alone are not particularly powerful for deducing spectral types in this range (e.g., Cushing et al. 2008).

At later spectral types 3-4 $\mu \mathrm{m}$ spectra do exhibit distinct features compared to late M-dwarfs and early L-dwarfs because of increased methane opacity and decreased cloud opacity at cooler temperatures. The latest spectral type source in our sample, CD-35 $2722 \mathrm{~B}$, which is classified as L3 based on NIR spectral indicators (Allers \& Liu 2013), appears redder than the best fit L5 field dwarf spectrum, with most of our measurements below the dwarf spectrum at the blue end of the band, and most of our measurements above the dwarf spectrum at the red end. This example suggests the importance of L-band spectroscopy for objects with later spectral type than mid-L, where the first hints of $\mathrm{CH}_{4}$ absorption are expected to arise (Noll et al. 2000).

\section{DISCUSSION}

\subsection{Comparison to Directly Imaged Planets}

The low surface gravity prevalent in young planetary mass atmospheres is responsible for the presence of clouds and disequilibrium chemistry at effective temperatures $\sim 800$ to $1300 \mathrm{~K}$, where older more massive T-dwarfs appear cloud free and methane rich (Marley et al. 1996; Barman et al. 2011a; Marley et al. 2012; Skemer et al. 2012). Here we observe a warmer more-massive population of young companions. Most of our atmospheric fits are consistent with the fits found by previous authors using NIR spectra. The similarity of our L-band derived atmospheric parameters compared to near-IR results demonstrates the functionality of the $\mathrm{MagAO} / \mathrm{Clio} 2$ spectroscopic mode and the general success of model atmospheres to capture the most important physics responsible for the appearance of spectra at these effective temperatures, where clouds are expected and $\mathrm{CH}_{4}$ absorption is not.

Our data allow us to constrain the onset of peculiarities where young low-gravity objects appear to have L-band spectra distinct from field objects. This range must be below $\sim 1500 \mathrm{~K}$, the effective temperature found here for $\mathrm{AB}$ Pic $\mathrm{b}$ and $2 \mathrm{M} \mathrm{0103}(\mathrm{AB}) \mathrm{b}$. This interval of effective temperatures includes the L-T transition for field brown dwarfs, emphasizing that the discrepancies in 3-4 $\mu \mathrm{m}$ spectra may be related to the cloud clearing and chemical processes responsible for the evolution from L-dwarf to T-dwarf.

\section{2. $C D-352722 B$}

Allers \& Liu (2013) assigned a spectral type of L3 with intermediate surface gravity for CD-35 2722 B based on an average of several indicators. Those authors noted CD352722 B exhibited significantly higher gravity than 2MASS J03552337+1133437, which has similar infrared spectral type and age (Liu et al. 2013a). Among our targets, fits to BT-Settl models suggest that CD-35 2722 B exhibits the highest surface gravity, consistent with previous studies and the relatively old age of the object compared to the rest of our sample.

CD-35 2722 B was classified as an L4 by Wahhaj et al. (2011) based on the similarity of its J, H, and K-band spectra to 2MASS J2224438-015852, an object whose very red color is distinct from other field dwarfs and implies large amounts of atmospheric dust (Cushing et al. 2005; Stephens et al. 2009). CD-35 2722 B has a bluer NIR color than 2MASS J2224438- 

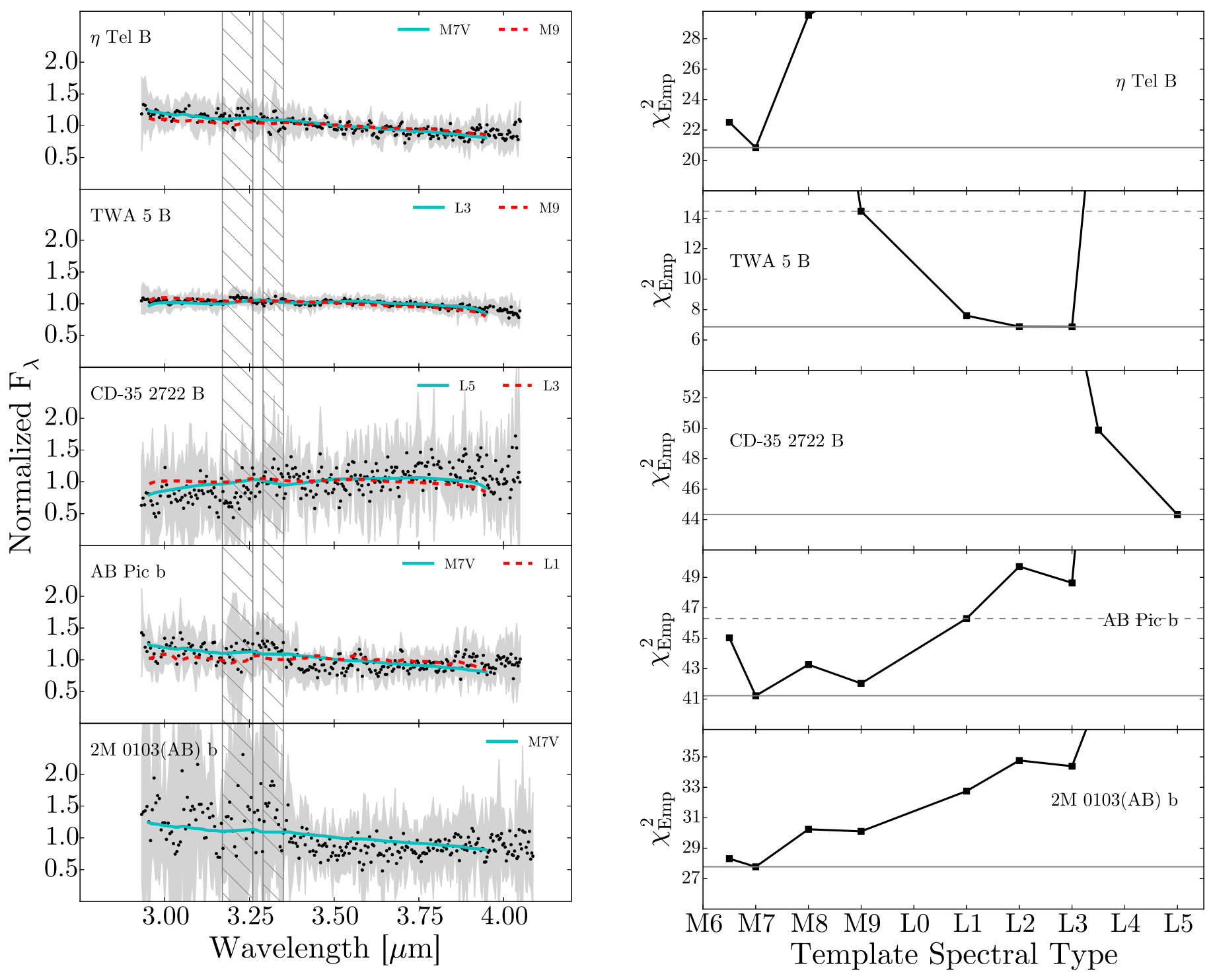

Figure 6. Left: the same companion spectra as in Figure 4, now compared to empirical spectra of field dwarfs from Cushing et al. (2005). With a solid cyan line we show the best fit field dwarf spectrum. We show the spectrum corresponding to the optical/NIR spectral type of each of our targets (Table 2) with a red dashed line. The field spectra for each spectral type correspond to the following targets: M7, GJ 644 C; M9, LP 944-20; L1, 2MASS J14392836+1929149; L3, 2MASS J15065441+1321060; L4.5, 2MASS J22244381-0158521; L5, 2MASS J15074769-1627386. Right: spectral type vs. $\chi^{2}$ resulting from our fitting analysis (solid black curves). The vertical extent of each plot shows the $99.7 \%$ confidence interval in $\Delta \chi^{2}$. We highlight the best fit model with a horizontal solid gray line. We also indicate the chi-square value of optical/NIR spectral type with a dashed line.

015852 (Wahhaj et al. 2011), suggesting somewhat different cloud structure.

Our spectrum of CD-35 2722 B is redder than the L5 field dwarf template spectrum we compared it to and best-fit model atmospheres are cooler than reported for NIR fits. These results are driven by a red slope to our spectrum. Since we do not have a telluric calibrator for the primary star in the CD-35 2722 system, we assumed the intrinsic shape of the star (based on its spectral type) when we calibrated the substellar companion. To check whether the red slope of CD-35 2722 B and our fitted parameters for this object could be due to selecting an overly red intrinsic spectrum for the primary, we repeated our telluric calibration procedure using a model corresponding to two spectral subtypes earlier than originally used. This did not result in a hotter best-fit model atmosphere.

CD-35 $2722 \mathrm{~B}$ is the oldest target in our young sample ( $\sim 50 \mathrm{Myr}$, Zuckerman et al. 2004), and has the highest surface- gravity. If its red 3-4 $\mu \mathrm{m}$ spectral slope does indicate thinning clouds, our observations suggest that this source may be cooler and of later type than previously thought, but this would need to be confirmed with higher signal-to-noise observations.

\section{3. $A B$ Pic $b$ and $2 M 0103(A B) b$}

As we pointed out in Section 3.1, the BT-Settl 2012 and 2015 grids provide very different best-fit effective temperatures for $A B$ Pic $b$ and $2 \mathrm{M} 0103(\mathrm{AB}) \mathrm{b}$. Each of these targets is better fit with a $1500 \mathrm{~K}$ model from the 2015 grid while fits to the 2012 grid show 2600 and $2900 \mathrm{~K}$ models are preferred for AB Pic b and 2M0103(AB) b, respectively. However, models with different effective temperatures have different predicted total fluxes. For an age of $30 \mathrm{Myr}$, the BT-Settl evolutionary models predicts $\mathrm{L}^{\prime}$ absolute magnitudes of 8.7 and $7.5 \mathrm{mag}$ for 2600 and $2900 \mathrm{~K}$ effective temperatures, respectively (Baraffe et al. 2015). These are both significantly brighter than the 
absolute L' magnitudes reported by Delorme et al. (2013) for AB Pic b $(9.9 \pm 0.1)$ and $2 \mathrm{M} 0103(\mathrm{AB}) \mathrm{b}(9.5 \pm 0.1)$. According to the models, the observed fluxes correspond to objects with effective temperatues $<1700 \mathrm{~K}$.

Both $\mathrm{AB}$ Pic b and 2M 0103(AB) b exhibit an increase in flux in the spectral region between $\sim 2.9$ and $3.4 \mu \mathrm{m}$. While part of this range includes a region of strong telluric absorption which challenges precise calibration, a significant portion is outside the strongest telluric features. None of the atmospheric models predict such a feature and the high effective temperatures suggested by the BT-Settl 2012 fits are probably incorrect, caused by the average blue slope of the spectra due to the anomalous blue feature.

For $2 \mathrm{M} 0103(\mathrm{AB}) \mathrm{b}$, the feature may be due to a combination of lower signal-to-noise and difficulties in observing and calibrating associated with orbiting a close binary (e.g., reduced AO performance). However, for AB Pic b, we achieved higher signal-to noise across the spectrum. The spectrum of AB Pic b was corrected for telluric emission using the spectrum of $\mathrm{AB}$ Pic A, which was positioned within the slit with $\mathrm{AB}$ Pic $b$ and observed simultaneously; any atmospheric changes during the course of the observation should have been tracked perfectly by the primary. Furthermore, our model atmosphere fit for AB Pic A is exactly as expected for the spectral type (K1V; Torres et al. 2006; Pecaut \& Mamajek 2013), suggesting we have properly calibrated AB Pic A and identified the correct model for its intrinsic spectral shape when correcting $A B$ Pic b. Given the quality of the telluric correction of AB Pic A, we also checked whether correcting AB Pic b with the same A-star calibrator provided a different result. Regardless of whether HD 32007 or AB Pic A is used for telluric calibration, the increased flux short of $3.4 \mu \mathrm{m}$ persists. We also verified that the feature is present in both A-nods and B-nods independently and in the first half of our integrations as well as in the second half.

If the increase in our spectrum is physical, the larger-thanexpected flux in the spectral region corresponding to methane opacity could indicate an atmospheric inversion or possibly an aurora (e.g., Hallinan et al. 2015). However, strong $\mathrm{H}_{3}^{+}$ emission, often associated with aurorae (e.g., Brown et al. 2003), is not present at 3.5 and $3.7 \mu \mathrm{m}$. Polycyclic aromatic hydrocarbon molecules (PAHs) are known to have broad emission bands centered at 3.3 and 3.4 microns. However, fluorescent PAHs in the vicinity of $\mathrm{AB}$ Pic $\mathrm{b}$ and $2 \mathrm{M} \mathrm{0103}(\mathrm{AB}) \mathrm{b}$ are unlikely given the system ages. While AB Pic A is known as a faint EUV and X-ray source (Lampton et al. 1997), this seems unlikely to cause strong PAH excitation. Additional follow up observations of AB Pic b in the L-band are warranted to better understand the blue feature seen in our data.

\section{CONCLUSIONS}

We commissioned the L-band spectroscopic mode of Clio2 behind the MagAO system. We observed five young systems with directly imaged low-mass companions. The recovered spectra of primary stars match templates for their literature spectral types, confirming the fidelity of the observations. The spectra of companions are consistent with expectations based on fits to shorter wavelength data. This result constrains the temperature range where the L-band SEDs of young directly imaged planets begin to diverge from older field dwarfs to $T_{\text {eff }} \lesssim 1500 \mathrm{~K}$. This range includes the the $\mathrm{L}-\mathrm{T}$ transition for field dwarfs, providing further evidence that observed discrepancies are due to clouds and non-equilibrium $\mathrm{CO} / \mathrm{CH}_{4}$ chemistry due to vertical mixing. The L-band spectrum of CD$352722 \mathrm{~B}$ is redder than field dwarfs and suggests some cloud settling in this $\sim 50$ Myr old object but low signal to noise limits our ability to draw any firm conclusions. We also see an increased flux feature from 2.95 to $3.4 \mu \mathrm{m}$ in the spectra of $2 \mathrm{M} \mathrm{0103(AB)} \mathrm{b}$ and AB Pic b. The feature coincides with methane bandheads, and if physical, could indicate a thermal inversion. L-band spectroscopy with Clio 2 will be an important complement to ongoing surveys for directly imaged extrasolar planets that are conducting their searches at shorter wavelengths.

This work was supported by NASA Origins grant NNX11AK57G and NSF AAG grant 121329. J.M.S. was also partially supported by the state of Arizona Technology Research Initiative Fund Imaging Fellowship. A.S. and T.J.R. are supported by the National Aeronautics and Space Administration through Hubble Fellowship grants HST-HF251349 and HST-HF2-51366.001-A (respectively) awarded by the Space Telescope Science Institute, which is operated by the Association of Universities for Research in Astronomy, Inc., for NASA, under contract NAS 5-26555. K.M.M. was supported under contract with the California Institute of Technology, funded by NASA through the Sagan Fellowship Program. We thank M. Cushing for providing L-band spectra of field dwarfs.

\section{REFERENCES}

Allard, F., Homeier, D., Freytag, B., \& Sharp, C. M. 2012, in EAS Publications Ser., Vol. 57, ed. C. Reylé, C. Charbonnel, \& M. Schultheis (Les Ulis: EDP Sciences), 3

Allers, K. N., \& Liu, M. C. 2013, ApJ, 772, 79

Baraffe, I., Homeier, D., Allard, F., \& Chabrier, G. 2015, A\&A, 577, A42

Barman, T. S., Konopacky, Q. M., Macintosh, B., \& Marois, C. 2015, ApJ, 804,61

Barman, T. S., Macintosh, B., Konopacky, Q. M., \& Marois, C. 2011a, ApJ, 733, 65

Barman, T. S., Macintosh, B., Konopacky, Q. M., \& Marois, C. 2011b, ApJL, 735, L39

Beuzit, J.-L., Feldt, M., Dohlen, K., et al. 2008, Proc. SPIE, 7014, 18

Boley, A. C., Helled, R., \& Payne, M. J. 2011, ApJ, 735, 30

Bonnefoy, M., Chauvin, G., Lagrange, A.-M., et al. 2014, A\&A, 562, A127

Bonnefoy, M., Chauvin, G., Rojo, P., et al. 2010, A\&A, 512, A52

Boss, A. P. 1997, Sci, 276, 1836

Bowler, B. P., Liu, M. C., Dupuy, T. J., \& Cushing, M. C. 2010, ApJ, 723,850

Brown, R. H., Baines, K. H., Bellucci, G., et al. 2003, Icar, 164, 461

Burgasser, A. J., Simcoe, R. A., Bochanski, J. J., et al. 2010, ApJ, 725,1405

Chabrier, G., Johansen, A., Janson, M., \& Rafikov, R. 2014, in Conf. Proc. Protostars and Planets VI, ed. H. Beuther et al. (Tuscon: Univ. Arizona Press) 619

Chauvin, G., Lagrange, A.-M., Dumas, C., et al. 2004, A\&A, 425, L29

Chauvin, G., Lagrange, A.-M., Zuckerman, B., et al. 2005, A\&A, 438, L29

Close, L. M., Males, J. R., Morzinski, K., et al. 2013, ApJ, 774, 94

Currie, T., Burrows, A., Itoh, Y., et al. 2011, ApJ, 729, 128

Cushing, M. C., Marley, M. S., Saumon, D., et al. 2008, ApJ, 678, 1372

Cushing, M. C., Rayner, J. T., \& Vacca, W. D. 2005, ApJ, 623, 1115

Delorme, P., Gagné, J., Girard, J. H., et al. 2013, A\&A, 553, L5

Hallinan, G., Littlefair, S. P., Cotter, G., et al. 2015, Natur, 523, 568

Hinz, P. M., Rodigas, T. J., Kenworthy, M. A., et al. 2010, ApJ, 716, 417

Horne, K. 1986, PASP, 98, 609

Hubeny, I., \& Burrows, A. 2007, ApJ, 669, 1248

Konopacky, Q. M., Barman, T. S., Macintosh, B. A., \& Marois, C. 2013, Sci, 339, 1398

Kratter, K. M., Murray-Clay, R. A., \& Youdin, A. N. 2010, ApJ, 710, 1375

Lampton, M., Lieu, R., Schmitt, J. H. M. M., et al. 1997, ApJS, 108, 545 
Liu, M. C., Dupuy, T. J., \& Allers, K. N. 2013a, AN, 334, 85

Liu, M. C., Magnier, E. A., Deacon, N. R., et al. 2013b, ApJL, 777, L20

Lloyd-Hart, M. 2000, PASP, 112, 264

Lodato, G., Delgado-Donate, E., \& Clarke, C. J. 2005, MNRAS, 364, L91

Lord, S. D. 1992, A New Software Tool for Computing Earth's Atmospheric Transmission of Near- and Far-infrared Radiation, Technical Memorandum NASA-TM-103957, A-92154, NAS 1.15:103957 (NASA NTRS: Washington, D.C.)

Lowrance, P. J., McCarthy, C., Becklin, E. E., et al. 1999, ApJL, 512, L69

Lowrance, P. J., Schneider, G., Kirkpatrick, J. D., et al. 2000, ApJ, 541, 390 Luhman, K. L. 2012, ARA\&A, 50, 65

Macintosh, B., Graham, J. R., Barman, T., et al. 2015, Sci, 350, 6256.64

Marley, M. S., \& Robinson, T. D. 2015, ARA\&A, 53, 297

Marley, M. S., Saumon, D., Cushing, M., et al. 2012, ApJ, 754, 135

Marley, M. S., Saumon, D., Guillot, T., et al. 1996, Sci, 272, 1919

Marois, C., Macintosh, B., Barman, T., et al. 2008, Sci, 322, 1348

Marois, C., Zuckerman, B., Konopacky, Q. M., Macintosh, B., \& Barman, T. 2010, Natur, 468, 1080

Martin, E. L., Rebolo, R., \& Zapatero-Osorio, M. R. 1996, ApJ, 469, 706

Morzinski, K. M., Males, J. R., Skemer, A. J., et al. 2015, ApJ, 815, 108

Neuhäuser, R., Ginski, C., Schmidt, T. O. B., \& Mugrauer, M. 2011, MNRAS, 416, 1430

Neuhäuser, R., Guenther, E. W., Petr, M. G., et al. 2000, A\&A, 360, L39
Noll, K. S., Geballe, T. R., Leggett, S. K., \& Marley, M. S. 2000, ApJL, 541, L75

Öberg, K. I., Murray-Clay, R., \& Bergin, E. A. 2011, ApJL, 743, L16

Pecaut, M. J., \& Mamajek, E. E. 2013, ApJS, 208, 9

Pollack, J. B., Hubickyj, O., Bodenheimer, P., et al. 1996, Icar, 124, 62

Sivanandam, S., Hinz, P. M., Heinze, A. N., Freed, M., \& Breuninger, A. H. 2006, Proc. SPIE, 6269, 0

Skemer, A. J., Close, L. M., Szúcs, L., et al. 2011, ApJ, 732, 107

Skemer, A. J., Hinz, P. M., Esposito, S., et al. 2012, ApJ, 753, 14

Skemer, A. J., Marley, M. S., Hinz, P. M., et al. 2014, ApJ, 792, 17

Skemer, A. J., Morley, C. V., Zimmerman, N. T., et al. 2015, ApJ, 817, 166

Stephens, D. C., Leggett, S. K., Cushing, M. C., et al. 2009, ApJ, 702, 154

Thiabaud, A., Marboeuf, U., Alibert, Y., Leya, I., \& Mezger, K. 2015, A\&A, 580, A30

Torres, C. A. O., da Silva, L., Quast, G. R., de la Reza, R., \& Jilinski, E. 2000, AJ, 120,1410

Torres, C. A. O., Quast, G. R., da Silva, L., et al. 2006, A\&A, 460, 695

Wahhaj, Z., Liu, M. C., Biller, B. A., et al. 2011, ApJ, 729, 139

Webb, R. A., Zuckerman, B., Platais, I., et al. 1999, ApJL, 512, L63

Weinberger, A. J., Anglada-Escudé, G., \& Boss, A. P. 2013, ApJ, 762, 118

Yamamura, I., Tsuji, T., \& Tanabé, T. 2010, ApJ, 722, 682

Zuckerman, B., Song, I., \& Bessell, M. S. 2004, ApJL, 613, L65

Zuckerman, B., Song, I., Bessell, M. S., \& Webb, R. A. 2001, ApJL, 562, L87 